\title{
Prospective study of swallowing function in patients with cervical dystonia undergoing selective peripheral denervation
}

\author{
A Münchau, C D Good, S McGowan, N P Quinn, J D Palmer, K P Bhatia
}

\begin{abstract}
Objective-To characterise swallowing function in patients with cervical dystonia with botulinum toxin treatment failure, before and after selective peripheral denervation surgery.

Methods-Twelve patients with cervical dystonia had a thorough examination including standardised assessment for cervical dystonia, scoring of subjective dysphagia, and videofluoroscopic swallow. Videofluoroscopy was scored by consensus opinion between a speech and language therapist and an independent blinded radiologist using a validated scoring system.
\end{abstract}

Results-Seven patients with cervical dystonia experienced no subjective dysphagia either before or after surgery, although in all these patients there was objective videofluoroscopic evidence of underlying mild to moderate oropharyngeal dysphagia preoperatively and postoperatively. The most common finding was delayed initiation of swallow. Three other patients, also without subjective dysphagia before surgery, developed postoperative dysphagia. In these patients, videofluoroscopy showed a delayed swallow reflex before surgery, which was worse postoperatively in two. The remaining two patients had mild subjective dysphagia before surgery that improved postoperatively in one and deteriorated in the other. In the first, videofluoroscopy was normal preoperatively and postoperatively, and in the second, oral bolus preparation was moderately abnormal preoperatively and swallow initiation was delayed postoperatively. Mean subjective dysphagia scores did not change significantly. Apart from a significant improvement of tongue base retraction, videofluoroscopic scores were not significantly different after surgery. Postoperatively there was significant improvement of overall cervical dystonia severity and abnormal head rotation in the group as a whole. There was no correlation between age, duration of symptoms of cervical dystonia, preoperative or postoperative cervical dystonia severity, subjective dysphagia scores, or videofluoroscopic scores. However, in the five patients with persisting anterior sagittal head shift as part of the torticollis, tongue base retraction was less likely to improve after surgery compared with those without head shift.

Conclusion-Surgical denervation of dystonic neck muscles, leading to improved neck posture, can also improve tongue base retraction, which is a key component of normal bolus propagation. However, delayed swallow initiation, a common feature in patients with cervical dystonia, can be further compromised by surgery, leading to subjective dysphagia. In general, selective peripheral denervation seems to be a safe procedure with no major compromise of swallowing function.

(F Neurol Neurosurg Psychiatry 2001;71:67-72)

Keywords: Cervical dystonia, dysphagia, selective peripheral denervation

Cervical dystonia is characterised by abnormal head and neck postures brought about by involuntary sustained or repetitive neck muscle contractions. ${ }^{1}$ Medical treatment is helpful in only $20 \%-30 \%$ of cases and side effects are common. ${ }^{2}$ Botulinum toxin injections given locally into active muscles causing abnormal head postures have been used with great success. ${ }^{3-8}$ It is becoming clear, however, that about $5 \%-10 \%$ of patients with cervical dystonia do not respond sufficiently to botulinum toxin injections. For these patients surgery becomes an option.

Before the advent of botulinum toxin various operations had been tried. ${ }^{9}$ The McKenzie operation, developed in the $1920 \mathrm{~s},{ }^{10}$ involved bilateral $\mathrm{C} 1$ to $\mathrm{C} 3$ rhizotomies, but was associated with significant morbidity and mortality, ${ }^{9}$ without evidence of lasting benefit, ${ }^{11}$ and is therefore no longer recommended. The current operation of choice is selective peripheral denervation, which aims to denervate muscles causing abnormal movements, while preserving innervation to those not participating in the dystonia. ${ }^{12-19}$

Although cervical dystonia causes distress and social stigmatisation, ${ }^{20}$ it is not a life threatening condition itself. In view of the many potential candidates for surgery, operative hazards become an important issue. Dysphagia impairing the quality of life, interfering with nutrition, and possibly causing aspiration, is probably the most troublesome potential adverse effect of surgery. In a review of different surgical procedures including selective peripheral denervation and rhizotomy, the incidence of dysphagia has been reported to be as high as $30 \%$ postoperatively. ${ }^{21}$ 
Videofluoroscopy before and after rhizotomy has shown moderate or severe swallowing dysfunction postoperatively in a third of the patients. ${ }^{22}$ Selective peripheral denervation seems to be safer in this respect, but as yet no systematic study of swallowing function in patients undergoing this procedure has been carried out.

We therefore conducted a prospective study of swallowing function in patients with cervical dystonia undergoing selective peripheral denervation using standardised preoperative and postoperative clinical and videofluoroscopic assessment.

\section{Methods}

The study was approved by the joint medical ethics committee of the Institute of Neurology and the National Hospital of Neurology and Neurosurgery.

PATIENTS

We studied 12 patients with cervical dystonia with failure of botulinum toxin injection treatment who were referred for surgery. ${ }^{18}$ The interval between the last botulinum toxin injection and the assessment was at least 4 months in all patients. Treatment failure was defined as no or only marginal benefit after at least three consecutive sets of botulinum toxin injection. Two patients never had any significant benefit after botulinum toxin injections (primary treatment failure), and the remaining 10 had lost their initial favourable response to botulinum toxin after a mean of 6.4 years (SD 1.4) (secondary treatment failure). The male/ female ratio was $2: 10$, mean age 51 years (SD 15.7), mean age of onset 42.7 years (SD 13.7), and mean duration of symptoms 8.3 years (SD 2.1).

Four patients were not on any medication before or after surgery, four patients were taking oral benzodiazepines, two with unchanged regimens before and after surgery, and one each had reduced or terminated regimens after surgery. One patient was taking oral amitriptyline before and after surgery and one was taking amitriptyline, diazepam, and benzhexol before and amitriptyline and diazepam after surgery. One patient who was on benzhexol before surgery continued this medication, another continued to take voltarol.

ASSESSMENT OF PATIENTS

All patients were assessed clinically and videofluoroscopically before surgery and then again 3-6 months (mean 5 (SD 1.7)) after surgery. We did not study patients immediately after surgery to avoid non-specific perioperative impairment of swallow-for example, caused by intubation. The rationale for studying patients within 6 months after surgery was to avoid confounding effects of possible reinnervation of neck muscles, which is not expected to be of functional importance before 6 months. The interval between postoperative clinical and videofluoroscopic assessments was not greater than 2 weeks in any case.
CLINICAL ASSESSMENT

Cervical dystonia

On clinical examination the predominant type of cervical dystonia (torticollis, laterocollis, retrocollis, or anterocollis) was determined taking into account primarily the degree of restriction of head movements and the primary dystonic head position. For instance, a patient with rotation of the head to the right and tilt to the left was labelled right torticollis when left rotation was more restricted than right tilt, and vice versa. The degree of dystonic head rotation and head tilt was measured using a goniometer.

The severity was determined using the Toronto western spasmodic torticollis rating scale (TWSTRS), ${ }^{23}$ which includes assessment of the dystonic position of the head, neck, and shoulder, effectiveness of any sensory geste, how long the patient can keep the head in a straight position, and range of head and neck movement. The maximum severity score on this scale is 35 .

Dysphagia

The severity of subjective symptoms of dysphagia was rated according to Comella et al. ${ }^{24}$ Patients were instructed to indicate which of the following statements applied to them (ratings in parentheses): "I don't have swallowing problems" (0); "Occasionally bringing down the food is difficult, particularly with certain food, but I don't choke or cough" (1); "I often have difficulties with swallowing. I had to change my diet slightly, avoiding certain foods, and have occasionally choked or coughed" (2); I have difficulties with swallowing all the time and had to make major changes in my diet; I have lost weight and/or I choke and cough a lot while eating" (3). After completion of this questionnaire patients were additionally specifically asked whether they paid attention to cutting their food into small pieces and whether they habitually washed down foods with sips of fluids.

\section{VIDEOFLUOROSCOPY}

Videofluoroscopy was conducted using a Siemens Siremobil 2000 mobile C arm image intensifier. Patients were examined seated and the head position noted. Liquid barium was added to foodstuffs to make four barium textures which were presented to all patients: thin liquid, thick liquid, paste, and muffin. These were given to the patient in controlled volumes of between 5-15 ml. These boluses were given while the patient's head remained in the dystonic position-that is, patients were asked not to oppose dystonic neck muscle contractions and not to use their sensory geste. This was done because we were interested in studying the impact of surgery on swallowing function in the patients" "natural", dystonic neck position, in which they remain most of the time, rather than in a transiently corrected neck position. Both lateral and anterior-posterior views were taken. Preoperative and postoperative videofluoroscopy was performed and scored jointly by a speech and language therapist and a radiologist. Videos were presented in random order to a second, independent 
Table 1 Characteristics of patients with normal preoperative and postoperative subjective swallowing function (patients 1-7) and patients in whom subjective swallowing function changed after surgery (patients 8-12)

\begin{tabular}{|c|c|c|c|c|c|c|c|c|}
\hline \multirow[b]{2}{*}{ Patient } & \multicolumn{2}{|c|}{$\begin{array}{l}\text { Subjective } \\
\text { dysphagia }\end{array}$} & \multicolumn{2}{|c|}{$\begin{array}{l}\text { Oral bolus } \\
\text { preparation }\end{array}$} & \multicolumn{2}{|c|}{$\begin{array}{l}\text { Tongue base } \\
\text { retraction }\end{array}$} & \multicolumn{2}{|c|}{$\begin{array}{l}\text { Initiation of } \\
\text { swallowing reflex }\end{array}$} \\
\hline & Pre & Post & Pre & Post & Pre & Post & Pre & Post \\
\hline 1 & 0 & 0 & 0 & 0 & 1 & 0 & 2 & 2 \\
\hline 2 & 0 & 0 & 0 & 0 & 1 & 1 & 2 & 2 \\
\hline 3 & 0 & 0 & 1 & 1 & 2 & 1 & 2 & 3 \\
\hline 4 & 0 & 0 & 0 & 0 & 3 & 1 & 3 & 3 \\
\hline 5 & 0 & 0 & 3 & 1 & 3 & 1 & 3 & 2 \\
\hline 6 & 0 & 0 & 0 & 0 & 3 & 0 & 2 & 2 \\
\hline 7 & 0 & 0 & 0 & 0 & 1 & 1 & 2 & 2 \\
\hline 8 & 0 & 3 & 0 & 3 & 4 & 1 & 2 & 3 \\
\hline 9 & 0 & 1 & 0 & 1 & 0 & 1 & 3 & 2 \\
\hline 10 & 0 & 1 & 0 & 1 & 1 & 1 & 1 & 3 \\
\hline 11 & 1 & 0 & 0 & 0 & 0 & 0 & 0 & 0 \\
\hline 12 & 1 & 2 & 3 & 1 & 3 & 1 & 0 & 2 \\
\hline
\end{tabular}

Numbers represent scores as defined in the text. Higher scores indicate worse function.

blinded radiologist. Each scorer used a set scoring system (see appendix). Where there were any differences in scoring, a consensus opinion was reached with the speech and language therapist/first radiologist and the second, independent blinded radiologist.

The examination also received a global rating of "mild", "moderate", "marked", or "severe" if one or more dimensions was rated as $1,2,3$, or 4 respectively.

SURGICAL PROCEDURE

A modified Bertrand operation was carried out. ${ }^{12-1518}$ Surgical options are a selective denervation of the sternocleidomastoid muscle and levator scapulae muscle (by sectioning branches of the accessory nerve supplying the sternocleidomastoid and the dorsoscapular nerve, respectively) and/or sectioning posterior rami of the $\mathrm{C} 1-\mathrm{C} 5 / 6$ roots. The second is referred to as a posterior ramisectomy and results in a complete motor and sensory denervation of the corresponding posterior neck segments.

\section{STATISTICAL ANALYSIS}

For comparison of two related samples (before and after surgery) a paired samples $t$ test was used for normally distributed interval data and Wilcoxon signed ranks test for ordinal data. To measure a possible correlation between different variables, Spearman's rank order correlation coefficient was used. Comparison of nominal data was performed with a $\chi^{2}$ test (Fisher's exact test for small sample sizes). For all statistical analyses an adjusted p value $<0.05$ was considered to be significant.

\section{Results}

Of the 12 patients, seven reported no symptoms of swallowing dysfunction either before or after surgery (table 1). However, four of these patients, when asked about their eating habits, said they were used to cutting their food into small pieces and/or had a drink with their meal. Videofluoroscopy disclosed abnormalities, especially delayed swallowing initiation, before and after surgery in all these seven patients (table 1).

Three patients (8-10) who did not report subjective dysphagia before surgery reported worsening of their swallowing function postop- eratively (table 1 ). One of these patients already had to cut his food into small pieces to avoid swallowing difficulties before surgery and this became a necessity in the other two patients after surgery. All three had videofluoroscopic abnormalities of swallowing in the form of delayed initiation of the swallowing reflex $(n=3)$ or abnormal tongue base retraction $(n=2)$ before surgery (table 1$)$. The delayed swallow initiation deteriorated postoperatively in two of them and improved in one. Pre-existing abnormal retraction of the tongue base improved in one patient and remained unchanged in the other. In the third patient it became mildly abnormal. Oral bolus preparation deteriorated in all three patients.

Two patients reported mild dysphagia before surgery (table 1). Both were used to eating their food in small chunks and needed a drink to wash down the food. In one (patient 11) preoperative videofluoroscopy was normal, in the other (patient 12) there were abnormalities in oral bolus preparation and tongue base retraction. Subjective swallowing function normalised and videofluoroscopy remained normal after surgery in patient 11 . In patient 12 the subjective score deteriorated, which can be explained by the postoperative development of a delayed swallowing initiation evident on videofluoroscopy in this patient.

The four patients with postoperative subjective dysphagia (patients 8-10, and 12) had more difficulty swallowing solid food than liquids. Chunks of meat and bread produced the greatest difficulties, often requiring several volitional swallows to clear the throat. The most severely affected patient (patient 8) required a change to a liquid diet for 6 weeks, without weight loss.

In one patient pharyngeal-oesophageal segment opening was mildly abnormal before but not after surgery. In another patient it was normal before but became mildly abnormal after surgery. In all the other patients it was normal preoperatively and postoperatively. Laryngeal penetration of the bolus was not found in any patients before and in one patient after surgery (score of 1).

Mean scores of all patients for severity of cervical dystonia, subjective dysphagia, and videofluoroscopy scores for the oral and pharyngeal phase of the swallowing before and after surgery are given in table 2 . Postoperatively there was a significant improvement of overall severity of cervical dystonia and abnormal head rotation, and a non-significant improvement of head tilt. Subjective dysphagia scores did not change significantly. Apart from a significant improvement of tongue base retraction, videofluoroscopic scores, including the global rating, were not significantly changed after surgery.

We did not find an overall correlation between subjective dysphagia scores and global videofluoroscopic rating or any other videofluoroscopic subscore before or after surgery. Also, changes in subjective and objective dysphagia scores were not correlated with each other. There was no correlation between age, duration of symptoms, preoperatively or post- 
Table 2 Severity of $C D$ and dysphagia before and after surgery

\begin{tabular}{|c|c|c|}
\hline & Before surgery & After surgery \\
\hline Overall severity* & $20.7(5.8) \dagger$ & $14.8(5.1)$ \\
\hline Head rotation & $50.4(31) \int$ & $26.5(22.6)$ \\
\hline Head tilt & $19.2(12.2)$ & $14.5(6.9)$ \\
\hline Subjective dysphagia score & $0.2(0.4)$ & $0.6(1)$ \\
\hline \multicolumn{3}{|l|}{ Videofluoroscopy: } \\
\hline Global rating & $2.3(1.1)$ & $2.5(0.5)$ \\
\hline Oral bolus preparation & $0.7(1.2)$ & $1.1(1.1)$ \\
\hline Tongue base retraction & $2.5(1.6) \ddagger$ & $1.4(0.9)$ \\
\hline $\begin{array}{l}\text { Initiation of swallowing } \\
\text { reflex }\end{array}$ & $1.8(1)$ & $2.2(0.8)$ \\
\hline Hyoid elevation & $1.9(1.7)$ & $1.3(1.7)$ \\
\hline Residue & $1.4(1)$ & $1.2(1.2)$ \\
\hline Symmetric swallow & 7 & 8 \\
\hline Asymmetric swallow & 5 & 4 \\
\hline
\end{tabular}

Apart from symmetry of swallowing all values are means (SD). ${ }^{\star}$ Determined by the TWSTRS; for dysphagia scores higher numbers indicate worse function.

$\dagger \mathrm{p}=0.017 ; \neq \mathrm{p}=0.027$ (Wilcoxon signed rank test).

$\mathrm{\rho}=0.034$ (paired samples $t$ test).

Significant improvements are indicated by bold numbers.

Table 3 Relation between postoperative improvement of tongue base retraction and postoperative head shift

\begin{tabular}{lll}
\hline & \multicolumn{2}{c}{ Sagittal head shift } \\
\cline { 2 - 3 } & Present & Absent \\
\hline Tongue base retraction & & \\
$\quad$ Improved after surgery & 1 & 6 \\
$\quad$ Unchanged or deteriorated after surgery & 4 & 1 \\
\hline $\mathrm{p}=0.045$ (Fisher's exact test). &
\end{tabular}

operative TWSTRS scores (or change of TWSTRS scores), degree of abnormal head rotation or tilt (or change in degrees), and preoperative or postoperative videofluoroscopic scores (or change of these scores).

However, there was a relation between head shift and tongue base retraction scores (table 3). Five patients had an anterior sagittal shift of the head that persisted or became more prominent after surgery, but in the other seven patients no such shift was present. In patients with persisting anterior head shift, tongue base retraction was less likely to improve after surgery than in those without such a shift (table 3).

\section{Discussion}

In line with previous reports 2225 objective videofluoroscopic abnormalities of swallowing were frequent in this group of patients with cervical dystonia, even before surgery. Compared with the results of Comella et $a l^{4}$ and Riski et $a l,{ }^{25}$ who found objective evidence of dysphagia in $22 \%$ and $51 \%$ of patients with cervical dystonia, respectively, the proportion of patients with objective dysphagia in this study (11/12) was high. The reason for this high prevalence, albeit usually mild or moderate, is not clear. Some of our patients were taking anticholinergic medication that may alter peristaltic function ${ }^{26-28}$ but the proportion was not higher than in the study of Comella et al. ${ }^{24}$ Previous botulinum toxin injection can compromise swallowing function due to local spread of the toxin, ${ }^{324}$ but this is also an unlikely explanation, as none of our patients had received botulinum toxin injections during the 4 months before the assessment. If swallowing problems occur after botulinum toxin injections they usually start about 2 days after the injection and last for 2-3 weeks but not for months. ${ }^{329} 30$

Only two of the 11 patients with abnormal preoperative videofluoroscopy complained of difficulty in swallowing - that is, subclinical dysphagia was common. Similar findings have been reported by Riski et $a l,{ }^{25}$ but the reason for subclinical dysphagia is not clear. It is possible that dysphagia was longstanding and patients had become used to their symptoms or adopted spontaneous compensatory strategies. For instance, when asked about eating habits, most patients with subclinical dysphagia reported that they were used to cutting their food into small pieces and taking a sip of fluid with each swallow to avoid the food getting stuck in the throat. Although this indicates disturbed swallowing, the patients did not perceive it as abnormal. Moreover, as all patients had botulinum toxin treatment failure and often had not received effective treatment for more than a year, their neck symptoms (mean score on the TWSTRS scale) were more severe than in patients with cervical dystonia still responding to botulinum toxin treatment. ${ }^{31}$ Neck symptoms may therefore have overshadowed the relatively subtle alteration in oropharyngeal function. ${ }^{25}$

Postoperatively none of the videofluoroscopic scores deteriorated significantly. However, three patients (8-10), who did not report swallowing problems before surgery, complained of mild to moderate dysphagia after surgery. Additionally, in one patient (12) mild pre-existing subjective dysphagia deteriorated and became moderate (score change from 1 to 2 ). In these four patients there were parallel postoperative changes on videofluoroscopy. The main findings were deterioration in swallow initiation in three patients $(8,10$, and 12) and worsening of oral bolus preparation also in three (patients 8-10).

For the delayed swallow initiation it has to be noted that such a delay was also found in 11 out of 12 patients before surgery, suggesting that it might be an intrinsic abnormality in cervical dystonia (see below). In patients with postoperative worsening of swallow initiation perhaps pulling of the anterior rami of cervical roots C1-C5 during preparation of posterior rami for denervation leads to disturbances of the nerve supply to muscles involved in the early stages of the automatic swallow. Although intraoperative pulling of nerve fibres is expected to cause neurapraxis, that lasts only days or a few weeks, subtle changes of sensorimotor control, outlasting the phase of the acute nerve damage, may nevertheless have occurred.

All the four patients with postoperative deterioration of swallowing function had subclinical dysphagia before surgery. It is possible that in these patients both a rapid change of swallowing function, to which they could not adequately adapt, and an improvement of neck symptoms (table 2), potentially rendering them more aware of their swallowing problems, contributed to the dysphagia becoming noticeable. 
Similar observations have been made after botulinum toxin injections. ${ }^{24}$

The preoperative videofluoroscopic data did not predict which patients were likely to develop subjective dysphagia after surgery. Indeed, the four patients with postoperative deterioration of subjective dysphagia had relatively few abnormalities before surgery (table 1). Nor did we find a correlation between change of subjective and objective dysphagia scores, which might be explained by the few patients (four) with subjective change of swallowing function. There was also no correlation between subjective and objective dysphagia preoperatively.

Two possible explanations have been proposed for the causes of dysphagia in patients with cervical dystonia: abnormal posture of the neck leading to asymmetry of the swallowing, and "neurogenic" causes leading to a delayed swallow initiation. ${ }^{25} 29$

The fact that tongue base retraction improved after surgery, when there was also a significantly improved head position, illustrates the importance of head posture for normal swallowing. Tongue base retraction occurs at an early stage of the pharyngeal phase of the swallowing, and is an important prerequisite for an orderly propagation of the food bolus. ${ }^{32}$ It is easy to see how an abnormally rotated or tilted head and dystonically overactive neck muscles might mechanically impair the retraction of the tongue and thus compromise swallowing. It is therefore not surprising that an improved head position (and a decrease of dystonic muscular activity) after surgical denervation also improved tongue base retraction. In patients with a prominent anterior sagittal head shift, tongue base retraction did not improve (table 3), which is probably explained by the fact that such a head shift is usually caused by dystonic activity of deep anterior neck muscles that are not denervated by posterior ramisectomy.

The most consistent finding for "neurogenic" causes of dysphagia in patients with cervical dystonia seems to be delayed initiation of swallowing, which did not improve after surgery. It was also not correlated with severity or duration of symptoms or patients' age, suggesting that it is rather a primary abnormality in cervical dystonia. The exact oropharyngeal stimuli for the initiation of the pharyngeal or automatic portion of the swallow are incompletely understood. ${ }^{33}$ These portions may be triggered by contact of the bolus and the tongue with the faucial arches, and also by deep receptors in the tongue base that are stimulated as the bolus and the tongue move posteriorly. ${ }^{34}$ In this respect it is interesting to note that abnormal sensory processing, particularly abnormal proprioceptive input, has been described in patients with dystonia. ${ }^{35-38}$ Although evidence of abnormal spindle activity in patients with dystonia as yet only comes from studies of limbs, it is perhaps not unreasonable to assume that similar abnormalities of sensory processing also affect other body regions, including the pharynx.
We conclude that selective peripheral denervation is a safe procedure in regard to swallowing function. Surgical correction of an abnormal head posture can improve tongue base retraction. The delayed swallow initiation, which is the most frequent abnormality in patients with cervical dystonia, and is possibly caused by abnormal sensory processing in dystonia, sometimes deteriorates.

AM was supported by the Ernst-Jung Stiftung für Wissenschaft und Forschung in Hamburg, Germany and the Eugen Brehm Bequest, UK. This study was supported by a grant from the Dystonia Society, UK.

\section{Appendix: Examples of the videofluoroscopy assessment rating scale (adapted from Logemann, ${ }^{39}$, Rosenbek $e t$ $a l,{ }^{40}$ and $\left.S_{\operatorname{cott}}{ }^{41}\right)$}

BOLUS PREPARATION/MASTICATION

$0=$ Normal; $1=$ slight weakness of tongue noticeable during manipulation of bolus; $2=$ reduced fine control of tongue and lateral tongue movement, slight weakness of the muscles of mastication; $3=$ reduced range and coordination of tongue movement and inability to lateralise material with tongue, reduced tongue elevation to hard palate, inability to align mandible for mastication and/or reduced mandibular range of motion, weakness of muscles of mastication; 4=inability to chew, mash or manipulate bolus.

INITIATION OF SWALLOW

$0=$ Swallow is triggered as the head of the bolus crosses the ramus of the mandible; $2=$ slight delay in initiation of swallow as the bolus passes tongue base and passes ramus of mandible; $2=$ swallow delayed to the valleculae; $3=$ swallow initiated when bolus head passes laryngeal vestibule or enters pyriform sinuses; $4=$ no swallow is initiated.

1 Fahn S, Marsden CD, Calne DB. Classification and investigation of dystonia. In: Marsden CD, Fahn S, eds. Movement disorders 2. London: Butterworth-Heinemann, 1987:33258.

2 Fahn S. High dosage anticholinergic therapy in dystonia. Neurology 1983;339:255-61.

3 Anderson TJ, Rivest J, Stell R, et al. Botulinum toxin treatment of spasmodic torticollis. F R Soc Med 1992;85:524-9.

4 Tsui JKC, Eisen A, Stoessl AJ, et al. Double-blind study of Tsui JKC, Eisen A, Stoessl AJ, et al. Double-blind study of
botulinum toxin in spasmodic torticollis. Lancet 1986;ii: $245-7$.

5 Greene P, Kang U, Fahn S, et al. Double-blind, placebocontrolled trial of botulinum toxin injections for the treatment of spasmodic torticollis. Neurology 1990;40:1213-8.

6 Moore AP, Blumhardt LD. A double blind trial of botulinum toxin "A" in torticollis, with 1 year follow up. $\mathcal{F}$ Neurol Neurosurg Psychiatry 1991;54:813-6.

7 Kessler KR, Skutta M, Benecke R. Long-term treatment of cervical dystonia with botulinum toxin A: efficacy, safety, and antibody frequency. German Dystonia Study Group. $\mathcal{F}$ Neurol 1999;246:265-74.

8 Jankovic J, Schwartz K. Response and immunoresistance to botulinum toxin injections. Neurology 1995;45:1743-6.

9 Maccabe JJ. Surgical treatment of spasmodic torticollis. In: Marsden CD, Fahn S, eds. Movement disorders 1. London: Marsden CD, Fahn S, eds.
Butterworth; 1981:308-14.

10 McKenzie KG. Intrameningeal division of the spinal asccessory and roots of the upper cervical nerves for the
treatment of spasmodic torticollis. Surgical Gynecology and treatment of spasmodic

11 Meares R. Natural history of spasmodic torticollis and effect of surgery. Lancet 1971;ii:149-51.

12 Bertrand CM. Selective peripheral denervation for spasmodic torticollis: surgical technique, results, and observations in 260 cases. Surg Neurol 1993;40:96-103.

13 Bertrand CM, Molina-Negro P. Selective peripheral denervation in 111 cases of spasmodic torticollis: rationale and results. Adv Neurol 1988;50:638-43.

14 Braun V, Richter HP. Selective peripheral denervation for the treatment of spasmodic torticollis. Neurosurgery 1994; 35:58-63.

15 Braun V, Richter HP, Schröder JM. Selective denervation for spasmodic torticollis: is the outcome predictable? $\mathcal{f}$ Neurol 1995;242:504-7.

16 Krauss JK, Toups EG, Jankovic J, et al. Symptomatic and functional outcome of surgical treatment of cervical dystonia. $\mathcal{F}$ Neurol Neurosurg Psychiatry 1997;63:642-8. 
17 Ford B, Louis ED, Greene P. Outcome of selective ramisectomy for botulinum toxin resistant torticollis. $\mathcal{f} \mathrm{Neurol} \mathrm{Neu}$ tomy for botulinum toxin resistant
rosurg Psychiatry 1998;65:472-8.

18 Münchau A, Palmer JD, Dressler D, et al. Prospective study of selective peripheral denervation for botulinum-toxin resistant patients with cervical dystonia. Brain 2001;124 769-83.

19 Münchau A, Bahlke G, Allen PJ, et al. Polymyography combined with time-locked video recording (video-EMG) for pre-surgical assessment of patients with cervical dystonia. Eur Neurol (in press).

20 Jahanshahi M, Marsden CD. Body concept, disability and depression in torticollis. Behavioural Neurology 1990;3. 117-31.

21 Colbassani HJ, Wood JH. Management of spasmodic torticollis. Surg Neurol 1986;25:153-8.

22 Horner J, Riski JE, Ovelmen-Levitt J, et al. Swallowing in torticollis before and after rhizotomy. Dysphagia 1992;7:

23 Consky EA, Lang AE. Clinical assessments of patients with cervical dystonia. In: Jancovic J, Hallet M, eds. Therapy with cervical dystonia. In: Jancovic J, Hallet M, eds. Therapy with

24 Comella CL, Tanner CM, DeFoor-Hill L, et al. Dysphagia after botulinum toxin injections for spasmodic torticollis: after botulinum toxin injections for spasmodic torticolis: 10.

25 Riski JE, Horner J, Nashold BS. Swallowing function in patients with spasmodic torticollis. Neurology 1990;40 1443-5.

26 Christensen J. Effects of drugs on esophageal motility. Arch Intern Med 1976;136:532-7.

27 Bramble MG, Cunliffe J, Dellipiani AW. Evidence for a change in neurotransmitter affecting oesophageal motility in Parkinson's disease f Neurol Neurosurg Psychiatry 1978; 4:709-12.

28 Dodds WJ, Dent J, Hogan WJ, et al. Effect of atropine on esophageal motor function in humans. Am F Physiol 1981; 240:290-6.
29 Whurr R, Bhatia KP, Masarei A, et al. The incidence and nature of dysphagia following botulinum toxin injections for torticollis: a prospective study of 123 patients. Fournal of Medical Speech and Language Pathology 1999;7:196-207.

30 Münchau A, Bhatia KP. Uses of botulinum toxin injection in medicine today. BMF 2000;320:161-5.

31 Brashear A, Lew MF, Dykstra DD, et al. Safety and efficacy of NeuroBloc (botulinum toxin type B) in type A-responsive cervical dystonia. Neurology 1999;53:143946.

32 Cunningham ET, Donner MW, Jones B, et al. Anatomical and physiological overview. In: Jones B, Donner MW, eds. Normal and abnormal swallowing. Imaging in diagnosis and therapy. New York: Springer, 1991:7-32.

33 Logemann JA. Dysphagia in movement disorders. Adv Neurol 1988;49:307-16.

34 Larson C. Neurophysiology of speech and swallowing. In Logemann J, ed. Seminars in speech and language. New York: Thieme Stratton, 1985:275-92.

35 Kaji R, Rothwell JC, Katayama M, et al. Tonic vibration reflex and muscle afferent block in writer's cramp. Ann Neurol 1995;38:155-62.

36 Tinazzi M, Priori A, Bertolasi L, et al. Abnormal central integration of a dual somatosensory input in dystonia. Evidence for sensory overflow. Brain 2000;123:42-50.

37 Grunewald RA, Yoneda Y, Shipman JM, et al. Idiopathic focal dystonia: a disorder of muscle spindle afferent processing? Brain 1997;120:2179-85.

8 Münchau A, Corna S, Gresty M, et al. Vestibulo-collic reflex and vestibulo-voluntary interaction in patients with spasmodic torticollis. Brain 2001;124:47-59.

39 Logemann JA. Evaluation and treatment of swallowing disorders. Texas: Pro.ED, 1998.

40 Rosenbek JC, Robbins JA, Roecker EB, et al. A penetrationaspiration scale. Dysphagia 1996;11:93-8.

41 Scott A. Bethlehem assessment scale for the videofluoroscopic assessment of swallowing. La Trobe, Australia: La Trobe University/Bethlehem Hospital, 1997.

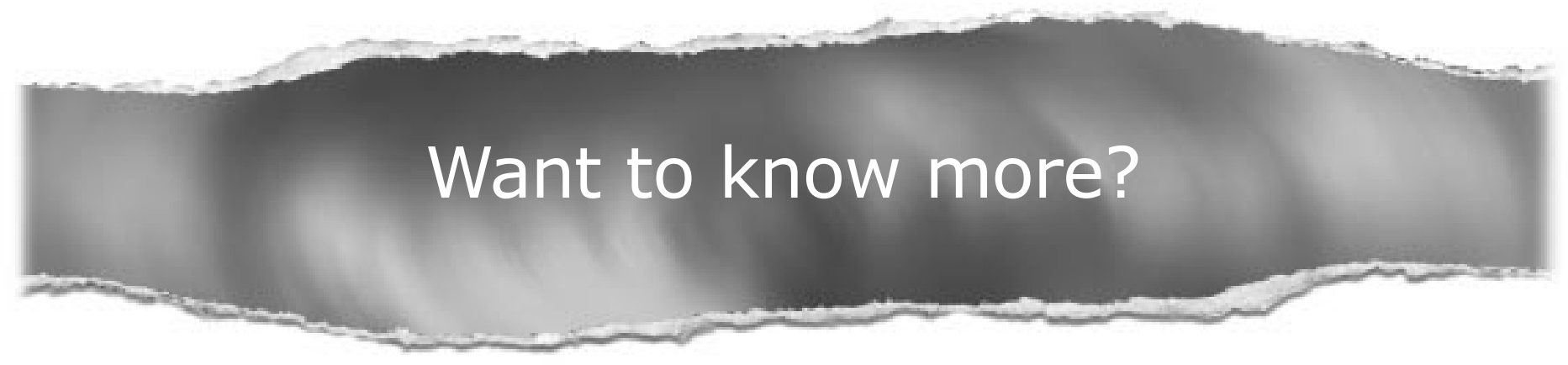

\section{Data supplements}

Limited space in printed journals means that interesting data and other material are often edited out of articles; however, limitless cyberspace means that we can include this information online.

Look out for additional tables, references, illustrations.

www.jnnp.com 